\title{
Quality Interactions in Early Childhood Education and Care Center Outdoor Environments
}

\author{
Karen L. Tonge ${ }^{1}$ [D $\cdot$ Rachel A. Jones ${ }^{1} \cdot$ Anthony D. Okely ${ }^{1}$
}

Published online: 21 August 2018

(C) The Author(s) 2018

\begin{abstract}
Quality interactions are crucial for children's learning and development. Early Childhood Education and Care (ECEC) centers have the opportunity to support children's learning and development, yet the quality of interactions and influences on the quality of interactions in outdoor environments is not known. Research findings: this study assessed the quality of educator interactions in outdoor environments using the CLASS Pre-K assessment tool. 11 ECEC centres participated in the study, which included 110 educators and 490 children. 87 observations were collected to measure the CLASS Pre-K domains (1-lowest to 7-highest). Mean domain scores were 6.02 (emotional support), 5.23 (Classroom Organization) and 4.46 (Instructional Support). Regression analyses show free routines had significant relationships with Teacher Sensitivity $(p=0.03)$ and Instructional Learning Formats $(p=0.03)$, and increased amounts of time spent outside had the most significant relationships with Teacher Sensitivity $(p=0.001)$ and Behavior Management $(p=0.001)$. Practice or Policy: The major recommendations that would serve to improve the quality of interactions in outdoor environments include providing a free routine and increasing the amount of time spent in outdoor environments. As these recommendations are modifiable practices, they are potentially the easiest to alter and therefore, with minimal change, could enhance the quality of interactions between educators and children.
\end{abstract}

Keywords Preschool $\cdot$ Interactions $\cdot$ CLASS Pre-K $\cdot$ Educators $\cdot$ Quality $\cdot$ Outdoor environments

\section{Introduction}

\section{The Early Years}

The early years (birth -5 years) are a time of rapid growth, including significant physical, cognitive, social-emotional and brain development (Shonkoff 2014). It is a time of opportunity where children's health and wellbeing, as well as quality experiences are an investment in learning and development (Shonkoff 2014). During these early years, many children attend an Early Childhood Education and Care (ECEC) center. In Australia, for example, $89 \%$ of

Karen L. Tonge

ktonge@uow.edu.au

Rachel A. Jones

rachelj@uow.edu.au

Anthony D. Okely

tokely@uow.edu.au

1 Early Start, Faculty of Social Sciences, University of Wollongong, Wollongong, NSW 2520, Australia children aged 4 years attend an ECEC center, and 92\% of these children attend for more than $15 \mathrm{~h}$ a week (ABS 2016). Furthermore, in most developed countries over the last two decades there has been an increase in children's attendance in formal ECEC experiences (OECD 2014). As such, ECEC centers play a critical role in the early life experiences for many children and are fundamental for children's learning and development, health and wellbeing.

\section{Early Childhood Education and Care Centers}

ECEC centers support children's learning and development through the provision of quality physical and social environments. This includes ensuring the availability of adequate equipment and space, as well as opportunities for structured and unstructured experiences and interactions (Ward 2010). Educators have a significant role in these ECEC environments as they facilitate experiences, and provide opportunities to engage in interactions with children. Establishing quality interactions between children and educators is crucial (DEEWR 2009; Ritchie and Howes 2003; Wang et al. 
2016) just as quality physical environments are for children's learning and development.

ECEC centers typically provide indoor and outdoor environments, and educators are encouraged to place equal value on these environments as places for children's learning and development (NQS 2016). Both environments offer opportunities for children and provide experiences in all developmental areas. While there may be variation in the features and proportion of time spent in each environment, the quality of experiences and interactions that occur in these environments are equally significant (NQS 2016). Despite the importance of both environments to a child's development, little is known about the influence of an educator's interactions with children in outdoor environments; consequently, the value of the outdoor environment for learning and development may be undervalued (Ulset et al. 2017). The opportunities that outdoor environments provide-such as space, natural playscapes and access to equipment (e.g., bikes, climbing equipment and balls) also reinforces their unique role in children's learning, health, and development.

\section{Outdoor Environments in ECEC Centers}

All ECEC centers worldwide offer an outdoor environment, or an environment that replicates one. For ECEC centers in Australia, the provision of an outdoor environment is a requirement of the National Quality Standards (NQS 2016). Typically, outdoor environments in ECEC centers provide many opportunities for children, including experiences that are unique to the space, such as building gardens, playing with trees and sandpits and playing in large open areas. The actual use of the outdoor space is managed at a center level, as is the proportion of the day that children have access to this environment. Some ECEC centers provide free flowing routines where children select the environment that they play in (i.e., children can choose to be the indoor environment or the outdoor environment at any point throughout the day), whereas other centers regulate the use of the particular environment at various times of the day, including what occurs within the environment at that time, such as a group experience. Educators utilize and prepare the space for various educational and recreational purposes that support children's learning and development, including the promotion of gross motor skills; experiences such as painting, reading and building that may also be present indoors; and activities that may not be possible or ideal indoors, such as bike riding and ball games. Research has shown that children's physical activity is greater in outdoor environments than in indoor environments (Tandon et al. 2015), reinforcing its importance in promoting active lifestyles.

Although it is clear that outdoor environments provide valuable opportunities for children's learning and development, much less is known about what happens in these environments compared with indoor environments. In particular, there are no known studies that have examined the quality of an educator's interactions with children in outdoor environments. This is important given that children will typically spend up to $9 \mathrm{~h}$ each day in these environments (Ulset et al. 2017) and that these environments are mandated in Australia in the NQS (2016).

\section{Quality in Early Childhood Education and Care Centers}

Improved outcomes for children in ECEC centers often are associated with the quality of the learning environment (Howes et al. 2008; Mashburn et al. 2008; Sylva et al. 2006). Although perspectives of quality in ECEC vary, research on quality has typically focused on structural characteristics, such as teacher-child ratios, group sizes and level of teacher education (LaParo et al. 2012). An alternative, yet equally important focus, is the quality of processes, such as interactions and engagement between educators and children (Howes et al. 2008). The study of process quality has shown that children's interaction and engagement with educators is related to their achievements (Burchinal et al. 2008; Cameron et al. 2005), and that quality interactions are the foundation of educators being powerful role models for children (Goldfield et al. 2012). In light of the importance of quality interactions for children's achievements, it is crucial to measure process quality in all learning environments, including outdoor environments. Additionally, it is crucial to measure process quality in light of ECEC center practices, such as routines and time spent in environments, as these may influence the quality of environments and interactions.

\section{Assessment of Quality in Early Childhood Education and Care Centers}

Many instruments measuring quality in ECEC centers have assessed multiple aspects, both structural and process (Byrant 2010) and although many of these instruments measure relevant components of the learning environment, the focus is more on processes such as physical and organizational structure (LaParo et al. 2004). Instruments such as the Classroom Assessment and Scoring System (CLASS) Pre-K (Pianta et al. 2008) offer a specific measure of the quality of interactions between educators and children. CLASS Pre-K is a real-time observational tool that assesses the quality of interactions between educators and children in ECEC environments based on specific and focused observations of individual educators. Central to CLASS Pre-K is the theoretical framework that educator and child interactions are crucial for academic and social-emotional success (Sandilos et al. 2014). The assessment is based on three core domains of interactions: emotional support, classroom 
organization and instructional support. Although predominantly used for assessment in US classrooms, CLASS Pre-K has been validated across a range of classrooms, for example, in ECEC centers with diverse languages (Downer et al. 2010), in various countries (Pakarinen et al. 2010; Tayler et al. 2016) and in comparison to other assessments of quality such as ECERS (LaParo et al. 2004). Findings indicate that CLASS Pre-K operates consistently across centers, demonstrating that it could function as a tool for improving quality in ECEC centers (Pianta et al. 2008). Despite the validation of CLASS Pre-K in various ECEC centers, a limitation of these studies is that the specific ECEC environment (indoor and/or outdoor) has not been identified. The use of CLASS Pre-K solely in outdoor environments in this study extends our understanding of CLASS Pre-K. Being aware of specific aspects of the quality of educator and child interactions, as well as possible influences on these interactions has the potential to empower educators to facilitate practices that support learning and development, health and wellbeing outcomes for children.

\section{The Current Study}

As outdoor environments and quality interactions are important for children's learning and development, understanding factors such as how the indoor-outdoor routine and the time spent outdoors influence the quality of interactions in outdoor environments will make an important contribution to optimising children's learning and development in ECEC centres. Therefore the aims of this study were to:

1. Report on CLASS Pre-K scores in ECEC centre outdoor environments, and to

2. Examine how the indoor-outdoor routine and the amount of time spent outdoors are related to CLASS Pre-K scores in ECEC center outdoor environments.

\section{Materials and Methods}

\section{Early Childhood Education and Care Centers and Participants}

In 2015, 11 ECEC centers located within a radius of $100 \mathrm{~km}$ from Wollongong, NSW, Australia, were recruited. ECEC centers were eligible to participate if they enrolled children aged 2-5 years, and these children had access to outdoor play spaces which were separate from other play spaces for younger children in the center. All eligible children and educators were invited to participate in the study, irrespective of the number of days enrolled or employed, respectively. Information about the study was presented to educators and families at staff and parent meetings and all eligible educators and children were provided with Participant Information Sheets and Consent forms. The study included a range of centers with variations in: the routine of the day, size and features of the physical environment, the number of children enrolled, and the use of indoor and outdoor environments, including the time that children have access to these environments. The detailed methods for the study from which these data were drawn were described in a previous paper (Tonge et al. 2016).

\section{Observation Measure-CLASS Pre-K}

Observational data were collected from educators and children in the centers. The CLASS Pre-K assessment scale was used to measure the quality of interactions between educators and children in the outdoor environment. CLASS Pre-K is an observation based assessment for use in ECEC environments and provides a contextualised assessment of interactions based on real-life observations (Pianta et al. 2008). It was selected as the most suitable assessment as it measures the quality of interactions with a specific focus on educators.

CLASS Pre-K consists of 10 dimensions measuring three domains (emotional support, classroom organization and Instructional Support) of classroom quality. Each dimension was rated on a 7-point Likert-scale (LaParo et al. 2004): low $(1,2)$, moderate $(3-5)$, or high $(6,7)$ according to the CLASS Dimensions Overview, Pre-K-3 document (Pianta et al. 2008). The dimensions in the emotional support domain focus on the interactions that support social and emotional functioning in the environment, such as positive communication and expectations; responsiveness; and providing children with responsibilities and freedom of movement. These social and emotional attributes support motivation and connectedness to the learning environment (Hamre and Pianta 2001; Silver et al. 2005), essential for children's learning and development. The classroom organization domain includes dimensions that relate to environment processes, such as an educator's organization and management of behavior, time and attention (Emmer and Stough 2001), as well as effective questioning, use of resources and clarity of objectives. When these situations are well managed, learning environments function effectively and provide optimal conditions for children to engage in experiences for learning. The dimensions in the instructional support domain are based on the processes of children's acquisition of knowledge and the implementation of experiences, such as problem solving; prediction and experimentation; real life application; teacher scaffolding; and effective conversations. In particular, this domain identifies cognitive and language development as key to child outcomes, and as with the other CLASS domains, quality interactions between children and educators as essential for children's learning and development in ECEC centers. 


\section{Observation Protocol}

Data were collected from outdoor environments in each ECEC center across five consecutive days. Throughout the data collection period, educators who were present in the outdoor environment were selected to be observed. To ensure a range of educators from each ECEC center were observed, when there was more than one educator in the outdoor environment, educators who had not been observed previously were selected.

The frequency and timing of observations varied between centers, and were dependent on the center routine and presence of children in the outdoor environment. The CLASS system has been validated for use in coding video recordings (Mashburn et al. 2008) and thus all observations in the study were video recorded using a portable video recorder and scored retrospectively. To ensure the recording adequately captured all auditory information, the educator being observed wore a bluetooth microphone which transmitted all sounds in proximity of the educator, including verbal interactions. To ensure accuracy in the visual information collected, the researcher remained close to the observation area, as discretely as possible.

Recording the observations allowed for greater measurement scrutiny and more accurate scoring between the two observers. This was especially important when there was uncertainty in the observations, allowing for cross-checking between observers. The process of recording observations was also important as outdoor environments in ECEC centers are typically larger than indoor environments and additional noise, obstacles and limited proximity to the event may occur. Recording observations ensured all aspects of the interactions (verbal and nonverbal) were able to be observed and assessed, even if the researcher was recording from a distance.

Observations met the criteria for CLASS scoring if they were more than $10 \mathrm{~min}$ in duration (Pianta et al. 2008) and the visual and auditory quality was satisfactory. At times the educator being observed completed tasks other than interactions with the children, including administration, programming and/or interactions with other educators and parents. These observations were still eligible for scoring as they provided insight into various influences on educator and child engagement and interactions.

During the observation period prior to scoring, observers made detailed notes about the CLASS Pre-K indicators. Immediately following the observation period, notes from each of the indicators were reviewed and based on these, scores from the CLASS Pre-K range (1 - lowest to 7 - highest) for each dimension were recorded on the CLASS Pre-K scoring sheet (Pianta et al. 2008). For each item the ratings were averaged across all cycles to produce the final score for the domain. For all domains, except the negative climate, the higher the score, the more positive the interaction. The dimension negative climate was reversed scored as per the CLASS Pre-K manual (Pianta et al. 2008).

\section{Training}

Prior to scoring the recorded observations, two researchers participated in preliminary training. An online training package 'Introduction to the CLASS Tool' (Teachstone Training LLC (C) consisting of five modules, approximately $30 \mathrm{~min}$ each in duration, was completed. This online package consisted of an overview of the purpose and structure of the CLASS tool as well as guided practice observation tasks that included observing an interaction, followed by multiplechoice questions to reinforce key elements of the interaction.

The second stage of training involved face-to-face professional development and consultation with other researchers, academics and practitioners who had used the CLASS Pre-K in their study. This one-day intensive workshop delivered by a certified CLASS Pre-K assessor provided opportunities for sharing knowledge as well as the purpose and implementation of the CLASS Pre-K assessment tool in ECEC centers.

\section{CLASS Pre-K Interrater Reliability}

Twelve observations (14\%) were double-scored by independent and trained observers. Reliability was $82 \%$ of dimension scores within a score of 1 on the 7-point CLASS scale. Previous studies have maintained at least $80 \%$ reliability (Jamison et al. 2014; Sandilos et al. 2014).

\section{Study Size}

This study forms part of a larger study examining the physical activity and location of children and educators in an outdoor ECEC setting (Tonge et al. 2016). In this larger study it was important to recruit enough educators to investigate the relationships at a centre level, and to allow for clustering at the ECEC level based on an intraclass correlation of 0.01 and an average cluster size of 10 . Accordingly, approximately 85 educators were needed to be recruited for the main study (Tonge et al. 2016). To recruit at least 85 educators, 11 ECEC centers participated, on the basis of each ECEC center employing between 6 and 15 educators.

\section{Early Childhood Education and Care Centers- Factors Influencing Quality}

For this study, two modifiable factors were examined in relation to the CLASS: center routine and the amount of time spent outdoors each day (Table 1). The routine group included centers that offered either an indoor-outdoor program or an aspect of the day that was indoor-outdoor (ie, children were 
Table 1 Early Childhood Education and Care center descriptives

\begin{tabular}{lllll}
\hline Centre code & $\begin{array}{l}\text { Number of CLASS } \\
\text { observations }\end{array}$ & $\begin{array}{l}\text { Number of educators } \\
\text { observed }\end{array}$ & ECEC routine & $\begin{array}{l}\text { Time spent out- } \\
\text { doors each day (avg } \\
\text { hrs) }\end{array}$ \\
\hline 1 & 6 & 6 & Free & 5.5 \\
2 & 8 & 8 & Structured & 2.5 \\
3 & 7 & 4 & Free & 4 \\
4 & 4 & 4 & Structured & 2 \\
5 & 7 & 5 & Structured & 2 \\
6 & 10 & 8 & Free & 5.5 \\
7 & 11 & 7 & Structured & 3.5 \\
8 & 13 & 8 & Structured & 4 \\
9 & 7 & 4 & Free & 4 \\
10 & 8 & 5 & Structured & 2.5 \\
11 & 6 & 5 & Structured & 3 \\
\hline
\end{tabular}

able to freely move from the indoor environment to the outdoor environment and vice versa) or a structured routine, where children had designated times for indoor and outdoor experiences and there was no opportunity for free movement between the environments during the day. These were termed 'free routine' and 'structured routine' respectively. The time spent outdoors each day was based on the total time children and educators spent outdoors, as was collected from ECEC center directors and through direct observation.

\section{Statistical Methods}

CLASS scores for individual educators were entered into an Excel spreadsheet and the means, standard deviations and range of these scores were calculated. Using StataIC 13, adjustment was made for clustering of ECEC centers using the svyset command and linear regression analyses were performed to investigate the relationship between individual educator CLASS dimension scores $(n=87)$ and the ECEC center routine and time spent outside. Linear regression models were produced for each of the CLASS dimensions in each of the ECEC center groups $(n=2)$. Routine was classified as a categorical variable (free or structured) and adjustment was made for educator age and qualification in these linear regression analyses. Time spent outside was classified as a continuous variable, and similar to the routine analyses adjustment was made for educator age and qualification, but also for centre type (long day care or preschool) as the total length of the day offered to children enrolled differs between preschools and long day care centers.

\section{Results}

\section{Descriptive Statistics}

From 11 ECEC centers, 110 educators and 490 children aged 2-5 years were recruited. Four of the centers provided an indoor-outdoor program and seven of the centers provided a structured program (Table 1). On one occasion the children were not present in the outdoor environment due to adverse weather and so the same day of the following week was scheduled for data collection.

\section{CLASS Pre-K}

A total of 131 observations were recorded. Two-thirds $(n=87)$ of the observations recorded met the CLASS criteria for this study and included 64 educators. Videos that did not meet the criteria and the reasons for this were: 23 videos (18\%) less than 10 min (these included educators leaving the environment due to commencing their lunch break, programming time, finishing their shift or all children moving inside), 14 videos (11\%) did not have clear audio and/or visual and seven videos (5\%) did not meet criteria for other reasons such as technical issues, a planned experience used for field notes or observation testing.

The average number of observations per center was eight (range 4-13) (Table 1). One CLASS observation was 
scored for $72 \%(n=46)$ of educators, and 18 educators were observed on multiple occasions. Two CLASS observations were scored for $20 \%(\mathrm{n}=13)$ of educators, and three observations were scored for $8 \%(n=5)$ of educators.

The educators were almost entirely female $(97 \%, \mathrm{n}=62)$ and the mean age was 35 years, with a range from 18 to 58 years of age. Educators reported a number of qualifications (16\% degree qualified, $42 \%$ diploma qualified, $31 \%$ certificate III qualified, $11 \%$ student) and numerous primary positions/responsibilities were reported (9\% Director, $2 \%$ Educational Leader, 3\% second in charge, $6 \%$ teacher, $28 \%$ advanced child care worker, $25 \%$ support, $11 \%$ casual, $11 \%$ student, $5 \%$ trainee).

Scores for CLASS domains and dimensions are described in Table 2. Mean scores were greatest in the emotional support domain and, from this domain, the dimension negative climate scored the highest $($ mean $=6.91)$. The lowest mean scores were in the instructional support domain, and in this domain, the dimension concept development scored the lowest overall (mean $=4.08)$. Using threshold values suggested by the CLASS measure (Pianta et al. 2008) these results suggest that across the 11 centers, emotional support was typically of high quality and classroom organization and Instructional Support were of medium quality.

\section{Linear Regression Analyses-CLASS Pre-K and Early Childhood Education and Care Center Factors}

A significant relationship was reported between free routines and teacher sensitivity $(\mathrm{p}=0.03)$ and instructional learning formats $(\mathrm{p}=0.03)$ (Table 3$)$. The relationship between free routine and concept development also approached statistical significance $(\mathrm{p}=0.06)$ (Table 3). In
Table 3 Relationship between Early Childhood Education and Care center routine and CLASS Pre-K dimensions

\begin{tabular}{lrll}
\hline & B coef. & $95 \%$ CI & $\mathrm{p}$ \\
\hline Emotional support & & & \\
Positive climate & -0.35 & $-0.95,0.26$ & 0.23 \\
Negative climate & 0.10 & $-0.05,0.25$ & 0.17 \\
Teacher sensitivity & -0.93 & $-1.72,-0.14$ & 0.03 \\
$\quad$ Regard for student perspectives & -0.43 & $-1.20,0.34$ & 0.25 \\
Classroom organization & & & \\
Behavior management & -0.56 & $-1.24,0.13$ & 0.10 \\
Productivity & -0.67 & $-1.56,0.21$ & 0.12 \\
Instructional learning formats & -0.92 & $-1.69,-0.14$ & 0.03 \\
Instructional support & & & \\
Concept development & -1.09 & $-2.22,0.05$ & 0.06 \\
Quality of feedback & -0.82 & $-1.86,0.22$ & 0.11 \\
Language modelling & -0.72 & $-1.72,0.29$ & 0.14 \\
\hline
\end{tabular}

Free is both indoor and outdoor environments available to children for all or some of the day. Structured is only selected environments (indoor or outdoor) are available to children

all of these cases, higher CLASS scores were reported when free routines were provided.

In the linear regression analysis for the time spent outdoors each day and CLASS dimensions (Table 4) significant relationships were reported for regard for student perspectives and teacher sensitivity $(\mathrm{p}=0.03$ and $\mathrm{p}=0.001$ respectively); instructional learning formats and behavior management ( $\mathrm{p}=0.01$ and $\mathrm{p}=0.001$, respectively); and concept development $(p=0.01)$. For each Item, higher CLASS scores were reported when more time was offered in the outside environment.

Table 4 Relationship between time spent outdoors each day and CLASS Pre-K dimensions

\begin{tabular}{lrrl}
\hline & B coef. & \multicolumn{1}{l}{$95 \%$ CI } & $\mathrm{p}$ \\
\hline Emotional support & & & \\
$\quad$ Positive climate & 0.15 & $-0.03,0.34$ & 0.10 \\
Negative climate & -0.03 & $-0.07,0.01$ & 0.09 \\
Teacher sensitivity & 0.39 & $0.19,0.59$ & 0.001 \\
$\quad$ Regard for student perspectives & 0.29 & $0.04,0.54$ & 0.03 \\
Classroom organization & & & \\
$\quad$ Behavior management & 0.35 & $0.19,0.51$ & 0.001 \\
$\quad$ Productivity & 0.35 & $-0.39,0.74$ & 0.07 \\
$\quad$ Instructional learning formats & 0.39 & $0.12,0.66$ & 0.01 \\
Instructional support & & & \\
$\quad$ Concept development & 0.49 & $0.18,0.79$ & 0.01 \\
Quality of feedback & 0.36 & $-0.11,0.84$ & 0.12 \\
Language modelling & 0.27 & $-0.10,0.65$ & 0.14 \\
\hline
\end{tabular}

\begin{tabular}{ll}
\hline CLASS dimensions & M (range, SD) \\
\hline Emotional support domain & \\
Positive climate & $6.28(2-7,0.11)$ \\
Negative climate $^{\mathrm{a}}$ & $6.91(6-7,0.03)$ \\
Teacher sensitivity & $5.53(2-7,0.14)$ \\
Regards for student perspectives & $5.34(2-7,0.13)$ \\
Classroom organization domain & \\
Behavior management & $5.89(3-7,0.10)$ \\
Productivity & $5.02(1-7,0.17)$ \\
Instructional learning formats & $4.78(1-7,0.17)$ \\
Instructional support domain & \\
Concept development & $4.08(1-7,0.18)$ \\
Quality of feedback & $4.79(1-7,0.17)$ \\
Language modelling & $4.51(1-7,0.18)$ \\
\hline
\end{tabular}

${ }^{a}$ Negative climate reserved scored 


\section{Discussion}

The purpose of this study was to report on CLASS Pre-K scores in ECEC centre outdoor environments, and to determine the influence of routines and the amount of time offered in outdoor environments on the quality of interactions between educators and children. Key findings indicate that providing a free routine that enables children to select either the indoor or outdoor environment; and greater amounts of time spent outside improves the quality of interactions between educators and children in ECEC centre outdoor environments.

The measurement of the quality of interactions between educators and children in ECEC outdoor environments is important because spending time in high-quality outdoor environments is critical for children's learning and development (Siraj-Blatchford 2009). Most studies reporting results from CLASS Pre-K have been methodological. For example, validation studies (Downer et al. 2010; Pakarinen et al. 2010) or studies that have compared CLASS Pre-K with others instruments that assess quality (LaParo et al. 2004) or studies that assess the stability of interactions during the day (Curby et al. 2010). A few studies have focused on relationships between CLASS Pre-K and outcomes such as educational wellbeing and social development (Burchinal et al. 2008; Curby et al. 2009; Tayler et al. 2016) or assessed the relationship between CLASS Pre-K scores and service type (Tayler et al. 2013). These studies consistently found that higher quality interactions resulted in improved outcomes for children. Although each of these studies has provided valuable information about quality interactions, there has been an absence of studies using CLASS Pre-K in the outdoor ECEC environment.

\section{CLASS Pre-K in Outdoor Early Childhood Education and Care Center Environments}

In this CLASS Pre-K study of the outdoor environment, the emotional support domain achieved the highest scores, and the instructional support domain achieved the lowest scores, a finding that is consistent with other CLASS Pre-K studies of indoor learning environments (Curby et al. 2010; LaParo et al. 2004; Sandilos and DiPerna 2011; Tayler et al. 2013). This outcome may be a reflection of an ECEC environment where children's social and emotional wellbeing is paramount and valued as being more crucial for learning and development than academic achievement. Educators advocate that children's learning will be optimised when they feel that they belong, and are supported, safe and secure (DEEWR 2009) - aspects assessed in the emotional support domain of CLASS Pre-K. Furthermore, in a study that measured the relationship between CLASS Pre-K emotional support domain scores and teacher efficacy, educators felt comfortable in a nurturing role, which aligns with indicators in the emotional support domain, such as sensitivity and creating a positive environment (Pakarinen et al. 2010).

Alongside the consideration that educators place high value on aspects in the emotional support domain, indicators in this domain, such as verbal and physical affection and providing comfort and assistance, may be more instinctive for educators compared with indicators in the instructional support domain, which scored the lowest. The instructional support domain relies on several skill-based concepts, such as advanced language, scaffolding, analysis and reasoning. Therefore, educators may require specific and intentional professional development to develop confidence in this domain. Accordingly, educators have indicated that they require further professional development to best support children's outcomes (Coleman and Dyment 2013; Tucker et al. 2011), and it may be this provision of professional development that results in higher instructional support domain scores.

The overall scores from CLASS Pre-K in this study indicate that the emotional support and classroom organization domains are in a high range of interaction quality, and that the instructional support domain is in the medium range. These ranges are higher than in other studies using CLASS Pre-K. For example, in other studies the mean scores for the emotional support and classroom organization domains were in the medium range, and the mean Instructional Support scores were in the low-medium range (Tayler et al. 2013; Sandilos and DiPerna 2011). Conversely, a study in Finland using CLASS (Pakarinen et al. 2010) found similar patterns to the current study with higher ranges reported. Possible explanations for this include the interpretation and evaluation of the dimensions; the absence of literature on CLASS Pre-K specifically in outdoor environments which has resulted in comparisons with indoor and/or outdoor rather than outdoor environments specifically; and the suitability of the CLASS Pre-K assessment in its entirety for outdoor environments which may have resulted in misrepresented scores. Further studies specifically in ECEC outdoor environments are needed to provide a more accurate comparison and interpretation.

The highest scores in the Emotional and lowest in the instructional support domain may have been influenced by the assessment being in the outdoor environment. Indicators in the instructional support domain suggest that highquality interactions are formed through defined exchanges, often requiring a high level of verbal interaction ("there are frequent conversations in the classroom' and 'the teacher often provides additional information to expand on students' understanding or actions'), whereas, in the emotional 
support domain, several indicators depend on non-verbal interactions ('there are frequent displays of positive affect by the teacher and/or students' and 'students have freedom of movement and placement during activities'). Affordances in outdoor environments differ from those in an indoor environment as the space is typically larger and opportunities for different experiences are available. For example, experiences that promote greater and faster movements such as climbing and bike riding are present, resulting in increased movement of and distances between educators and children. In these cases, measuring the quality of interactions by assessing verbal interactions may be compromised as the movement and location of educators and children may affect the level of verbal interactions that occur, as is linked to high-quality interactions in the instructional support domain. Interactions in outdoor environments may be more dependent on the educator's non-verbal involvement and interactions with children rather than verbal interactions. Subsequently this presents challenges in the assessment of the quality of interactions based on language modelling and conversations, as is indicated in the instructional support domain, more so than in the emotional support or classroom organization domains.

In addition to the suitability of the indicators of Instructional Support, the actions of the educators in this outdoor environment may influence the Instructional Support scores. Due to the specific features and affordances of an outdoor environment, such as gardens, climbing equipment, bikes and typically more active play, educators may perceive that their main role during outdoor play is the supervision and safety of children (Coleman and Dyment 2013). Consequently the outdoor environment may be underestimated as an intentional learning space. This perception may increase emotional support, to the detriment of instructional aspects such as concept development, effective feedback and language modelling (Pianta et al. 2008)—all indicators in the instructional support domain.

\section{The Relationship Between Quality of Interactions and Routines and Time Spent Outdoors}

ECEC centers are diverse and there are many factors, such as location, educator-child ratios, available space and resources (van Zandvoort et al. 2010), regulations and policies, as well as environmental factors such as the weather (Poest et al. 1989; Tucker and Gilliland 2007) that influence practice and therefore children's experiences and outcomes. These may have a greater influence in outdoor environments. ECEC centers may not have the capacity to manage all potential influences; however, it is evident in this study that there are factors, such as the type of routine and time spent outside, that educators can modify that may influence the quality of interactions between educators and children during time spent in outdoor environments.
When educators offered a free routine, such as children having access to indoor and outdoor environments at any time throughout the day, compared to a routine that was structured (e.g., children were indoors in the morning and outdoors in the afternoon) the quality of interactions between educators and children in an ECEC outdoor environment were consistently greater. Furthermore, other research has shown the benefits of a free routine that allows children to move freely between environments of choice on the amount of time children spend in experiences such as physical activity (Hesketh and van Slujis 2016). When children spend increased periods of time in experiences, this allows their play to extend and develop, and opportunities for sustained shared thinking (Siraj-Blatchford 2009), which are key aspects for learning and development, are increased. Enabling children to move freely between environments also allows children to make choices for their play, and therefore may have an influence on the quality of their play and interactions. Additionally, allowing children to move freely between environments of choice has the potential to minimise the number of children in each space, therefore ensuring resources and equipment are accessible, avoiding waiting times and conflicts that may arise. Identifying such influences on the quality of educator and child interactions is important to being able to design interventions that promote high quality environments.

Teacher sensitivity and instructional learning formats were related to both free routines and increased time spent outside. Teacher sensitivity focuses on awareness, responsiveness, addressing problems and student comfort (Pianta et al. 2008) whilst instructional learning formats focuses on effective questioning, teacher involvement and hands on opportunities. In an ECEC center when a free routine is provided, children have opportunities to move freely between environments, around peers, educators and experiences and potentially regulate their social and emotional experiences. In this emotional climate, children may be more comfortable and confident as they have a greater agency over their learning environment. Accordingly, the response of educators may reflect the disposition of the children within the environment, resulting in interactions that lead to more advanced motor skill development and opportunities for extended interactions. More time in an environment allows for these indicators to develop as transition times may be reduced, and children and educators have more opportunities to engage in sustained interactions (Siraj-Blatchford 2009).

Consistent results were also found when greater amounts of time were spent outdoors. When ECEC centers provided children with more time in the outdoor environment across the day, higher quality interactions were reported. Increased time in an environment allows sustained periods of time engaged in experiences, as well as reducing the 'novelty' factor that may occur when children 
have shorter periods of time in an environment. Sustained periods of time in an outdoor environment provides opportunities free from interruption due to transitions, preparation and packing away of equipment. Accordingly, sustained opportunities in experiences have the potential for higher-level engagement, challenge and problem solving (Siraj-Blatchford 2009) and subsequently environments that are stimulating (Melhuish 2004). These factors may have influenced the quality of the interactions in this study, as greater time allowed better quality environments to develop. Interestingly, other studies indicate that it is the quality of the time, and what occurs within experiences that is important for children's outcomes, such as physical activity (Dowda et al. 2004, 2009; Tonge et al. 2016). Recognising the influence of the quality as well as the quantity of the time spent outdoors is critical. The need for deliberate planning of time, experiences, interactions and intentional teaching in outdoor environments is essential and has the potential to influence the quality of interactions in the environment and subsequently child experiences and outcomes.

\section{Possibilities with CLASS Pre-K}

This was an exploratory study measuring each domain and dimension from CLASS Pre-K. Using the scale solely in outdoor environments was unique and has presented some areas for further consideration. The assessment of the quality of interactions in outdoor environments with CLASS Pre-K needs to consider the assessment scales and aspects of the items being measured. For example, the dimension productivity includes the criteria of maximising learning time and transitions. In an outdoor environment which is typically less structured, these aspects may not be as frequent. Additionally, due to outdoor environments in ECEC centers having a tendency to be more spontaneous, the clarity of learning objectives from the dimension instructional learning formats-as well as indicators in the classroom organization domain-may not be as pronounced. Future studies measuring the quality of interactions in outdoor environments need to consider possible misrepresentations of dimension scores and report according to the observed environment. As was suggested in a study using the inCLASS measurement tool (Downer et al. 2010), it is apparent that CLASS Pre-K has the potential to provide a contextualised assessment of educator and child interactions, one that may complement other ECEC center assessments. In the absence of any other appropriate tools for the outdoor environment, this assessment tool is currently the best choice and hence the reason it was used in this study.

\section{Strengths and Limitations}

This study has a number of strengths: (1) CLASS Pre-K assessed the quality of educator and child interactions in outdoor environments which has not been reported previously; and (2) identification of modifiable and achievable practices that support better quality interactions.

The focus on ECEC outdoor environments offers new information to what is already known about the quality of educator and child interactions in ECEC centers. The potential of outdoor environments as valuable learning spaces are often underestimated; therefore it is important to demonstrate the opportunities that they hold for children's learning and development. Further, it is important for educator and child interactions to be meaningful in ECEC center outdoor environments as this has the potential to enhance children's physical activity, physical activity promotion and skill development for children's health and wellbeing.

Identifying modifiable aspects of practice that educators have the ability to manage is empowering for educators. There are some aspects of ECEC centers such as the size of the yard, geographic location and number of children enrolled that cannot be modified, yet reviewing and modifying the routine provided and the amount of time spent outside are somewhat more achievable. As this study shows, these changes can have significant effects on the quality of interactions between educators and children, and therefore child outcomes.

The results of the study should, however, be considered in light of a number of limitations, including the limited observation time in some ECEC centers, and the design and nature of CLASS Pre-K being perhaps better suited for indoor than outdoor environments.

Although the CLASS manual (Pianta et al. 2008) suggests that the results are reflective of typical practice, this may be a limitation of the present study. The total observation time which is measured with CLASS Pre-K may not be representative of the quality of educator and child interactions throughout the day. In this study the collection of observations only in outdoor environments meant that not all educators were observed, and the timing of the observations was set to a timeframe, for example only when the children and educators were in outdoor environments. In some ECEC centers that offered a free routine, it was only selected educators that engaged in the outdoor environment, and although the observations were random, there were limitations as to which educators were observed. Additionally, a small number of educators chose not to be involved in the observations and recordings. In these free-routine ECEC centers, as educators and children had the potential to move between environments, this movement between environments sometimes would result in the observation 
ceasing. Further research comparing the quality of interactions between educators and children in outdoor and indoor environments is warranted.

ECEC center environments are diverse and features of ECEC center indoor and outdoor environments vary. Outdoor environments are typically larger and provide less structured experiences than indoor environments, and experiences may encourage more movement within and between areas, for example ball games, climbing equipment and portable equipment such as bikes and scooters. Consequently, children's and educators' movements may be different between these environments. It is apparent that the CLASS Pre-K tool has been designed for the indoor environment and previous studies using this tool may have only investigated the indoor environment. This warrants consideration of its application in outdoor environments. Central to CLASS Pre-K assessments are verbal interaction and as indoor environments are generally smaller environments it is easier to capture conversations, whereas in outdoor environments which are generally larger and more open this may be difficult. As such, it is paramount that observers utilise the most effective methods of capturing all verbal interactions within any environment without influencing typical practice. Observations in this study were video recorded allowing the movement of educator and children while still recording vital information. To ensure accuracy in audio information, the educator selected for the observation also wore a wireless microphone. This further improved clarity of audio data collected, particularly from a distance or while the educators were moving. To reduce the effects of wearing the microphone on typical practice, such as reactivity which may result in participating in additional interactions, or perhaps not as many interactions, multiple observations were collected across the period of data collection in the ECEC center.

\section{Conclusion}

High quality environments provide opportunities that support children's learning and development, and it is crucial that value is placed on both indoor and outdoor environments as opportunities to develop quality interactions. Recommendations for future research include further investigations into the influence of quality interactions in ECEC outdoor environments that will support all areas of children's learning, development, health and wellbeing. It is important that quality interactions are established to achieve positive outcomes and therefore it is important to understand potential factors that influence the quality of educator and child interactions in all environments. This study recommends that educators have the capacity improve the quality of interactions by considering modifiable practices and opportunities that are available. Providing an aspect of a free flowing routine each day where children can select to be indoors or outdoors, as well as increasing the amount of time spent outdoors, has shown a significant influence on quality educator and child interactions in outdoor environments. Consequently, establishing quality interactions throughout the ECEC environment has the potential to provide the best possible environments for children's learning, development, health and wellbeing.

Acknowledgements This research has been conducted with the support of the Australian Government Research Training Program Scholarship.

\section{Compliance with Ethical Standards}

Conflict of interest The authors declare that they have no conflict of interests.

Open Access This article is distributed under the terms of the Creative Commons Attribution 4.0 International License (http://creativeco mmons.org/licenses/by/4.0/), which permits unrestricted use, distribution, and reproduction in any medium, provided you give appropriate credit to the original author(s) and the source, provide a link to the Creative Commons license, and indicate if changes were made.

\section{References}

ABS, Australian bureau of statistics. (2016). 4240.0_Preschool education, Australia. http://www.abs.gov.au/ausstats/abs@.nsf/Lates tproducts/4240.0Main\%20Features12016?opendocument\&tabna me $=$ Summary \&prodno $=4240.0 \&$ issue $=2016 \&$ num $=\&$ view $=$.

Burchinal, M., Howes, C., Pianta, R., Bryant, D., Early, D., Clifford, R., et al. (2008). Predicting child outcomes at the end of kindergarten from the quality of pre-kindergarten teacher-child interactions and instruction. Applied Developmental Science, 12(3), 140-153.

Byrant, D. (2010). Observational measures of quality in centre-based early care and education programs. Research-to-Policy, Researchto-Practice Brief OPRE 2011-10c.

Cameron, C. E., McDonald-Connor, C., \& Morrison, F. J. (2005). Effects of variation in teacher organization on classroom functioning. Journal of School Psychology, 43(1), 61-85.

Coleman, B. D., \& Dyment, J. E. (2013). Factors that limit and enable preschool-aged children's physical activity on child care centre playgrounds. Journal of Early Childhood Research, 11(3), 203-221.

Curby, T. W., Grimm, K. J., \& Pianta, R. C. (2010). Stability and change in early childhood classroom interactions during the first two hours of a day. Early Childhood Research Quarterly, 25(3), 373-384.

Curby, T. W., LoCasale-Crouch, J., Konold, T. R., Pianta, R., Howes, C., Burchinal, M., et al. (2009). The relations of observed pre-K classroom quality profiles to children's achievement and social competence. Early Education and Development, 20(2), 346-372.

DEEWR, Australian Government Department of Education, Employment and Workplace Relations for the Council of Australian Governments. (2009). Belonging, being, becoming: The early years learning framework for Australia. Commonwealth of Australia.

Dowda, M., Brown, W. H., McIver, K. L., Pfeiffer, K. A., O’Neill, J. R., Addy, C. L., et al. (2009). Policies and characteristics of the 
preschool environment and physical activity of young children. Pediatrics, 123(2), 261-266.

Dowda, M., Pate, R. R., Trost, S. G., Almeida, M. J., \& Sirard, J. R. (2004). Influences of preschool policies and practices on children's physical activity. Journal of Community Health, 29(3), 183-196.

Downer, J. T., Booren, L. M., Lima, O. K., Luckner, A. E., \& Pianta, R. C. (2010). The individualized classroom assessment scoring system (inCLASS): Preliminary reliability and validity of a system for observing preschoolers' competence in classroom interactions. Early Childhood Research Quarterly, 25(1), 1-16.

Emmer, E. T., \& Stough, L. M. (2001). Classroom management: A critical part of educational psychology, with implications for teacher education. Educational Psychologist, 36(2), 103-112.

Goldfield, G. S., Harvey, A., Grattan, K., \& Adamo, K. B. (2012). Physical activity promotion in the preschool years: A critical period to intervene. International Journal of Environmental Research and Public Health, 9(4), 1326-1342.

Hamre, B. K., \& Pianta, R. C. (2001). Early teacher-child relationships and the trajectory of children's school outcomes through eighth grade. Child Development, 72(2), 625-638.

Hesketh, K., \& van Slujis, E. M. F. (2016). Features of UK childcare environment and associations with preschooler's in-care physical activity. Preventive Medicine Reports, 3, 53-57.

Howes, C., Burchinal, M., Pianta, R., Bryant, D., Early, D., Clifford, R., et al. (2008). Ready to learn? Children's pre-academic achievement in pre-Kindergarten programs. Early Childhood Research Quarterly, 23(1), 27-50.

Jamison, K. R., Cabell, S. Q., LoCasale-Crouch, J., Hamre, B. K., \& Pianta, R. C. (2014). CLASS-infant: An observational measure for assessing teacher-infant interactions in center-based child care. Early Education and Development, 25(4), 553-572.

La Paro, K. M., Pianta, R. C., \& Stuhlman, M. (2004). The classroom assessment scoring system: Findings from the prekindergarten year. The Elementary School Journal, 104(5), 409-426.

La Paro, K. M., Thomason, A. C., Lower, J. K., Kintner-Duffy, V. L., \& Cassidy, D. J. (2012). Examining the definition and measurement of quality in early childhood education: A review of studies using the ECERS-R from 2003 to 2010. Early Childhood Research \& Practice, 14(1), 1-13.

Mashburn, A. J., Pianta, R. C., Hamre, B. K., Downer, J. T., Barbarin, O. A., Bryant, D., et al. (2008). Measures of classroom quality in prekindergarten and children's development of academic, language, and social skills. Child Development, 79(3), 732-749.

Melhuish, E. C. (2004). Child benefits: The importance of investing in quality childcare. Facing the future: Policy papers no. 9. London: Daycare Trust.

National Quality Standards. (2016). Australian children's education and care quality authority. http://acecqa.gov.au/national-qualityframework/the-national-quality-standard.

Organisation for Economic Cooperation and Development, Family database. (2014). PF3.2: Enrolment in childcare and pre-schools. OECD—Social Policy Division-Directorate of Employment, Labour and Social Affairs.

Pakarinen, E., Lerkkanen, M.-K., Poikkeus, A.-M., Kiuru, N., Siekkinen, M., Rasku-Puttonen, H., et al. (2010). A validation of the classroom assessment scoring system in finnish kindergartens. Early Education and Development, 21(1), 95-124.

Pianta, C. R., LaParo, K. M., \& Hamre, B. K. (2008). Classroom assessment scoring system: Manual Pre-K. Baltimore: Paul.H. Brookes Publishing Co.

Poest, C. A., Williams, J. R., Witt, D. D., \& Atwood, M. E. (1989). Physical activity patterns of preschool children. Early Childhood Research Quarterly, 4, 367-376.
Ritchie, S., \& Howes, C. (2003). Program practices, caregiver stability, and child-caregiver relationships. Journal of Applied Developmental Psychology, 24(5), 497-516.

Sandilos, L. E., \& DiPerna, J. C. (2011). Interrater reliability of the classroom assessment scoring system - Pre-K (CLASS Pre-K). Journal of Early Childhood \& Infant Psychology, 7, 65-85.

Sandilos, L. E., DiPerna, J. C. \& The Family Life Project Key Investigators. (2014). Measuring quality in kindergarten classrooms: Structural analysis of the classroom assessment scoring system (CLASS K-3). Early Education and Development, 25(6), 894-914.

Shonkoff, J. P. (2014). Changing the narrative for early childhood investment. JAMA Pediatrics, 168(2), 105-106. https://doi. org/10.1001/jamapediatrics.2013.4212.

Silver, R. B., Measelle, J. R., Armstrong, J. M., \& Essex, M. J. (2005). Trajectories of classroom externalizing behavior: Contributions of child characteristics, family characteristics, and the teacherchild relationship during the school transition. Journal of School Psychology, 43(1), 39-60.

Siraj-Blatchford, I. (2009). Conceptualising proression in the pedagogy of play and sustained shared thinking in early childhood education: A Vygotskian perspective. Educational \& Child Psychology, 26(2), 77-89.

Sylva, K., Siraj-Blatchford, I., \& Taggart, B. (2006). Assessing quality in the early years: Early childhood environment rating scale extension (ECERS-E): Four curricular subscales. Revised ed. Stoke on Trent: Trentham Books.

Tandon, P. S., Saelens, B. E., Christakis, D. A., et al. (2015). Active play opportunities at child care. Pediatrics. https://doi. org/10.1542/peds.2014-2750.

Tayler, C., Cloney, D., Adams, R., Ishimine, K., Thorpe, K., \& Nguyen, T. K. C. (2016). Assessing the effectiveness of Australian early childhood education and care experiences: Study protocol. BMC Public Health, 16, 1.

Tayler, C., Ishimine, K., Cloney, D., Cleveland, G., \& Thorpe, K. (2013). The quality of early childhood education and care services in Australia. Australasian Journal of Early Childhood, $38(2), 13-21$.

Tonge, K. L., Jones, R. A., \& Okely, A. D. (2016). Correlates of children's objectively measured physical activity and sedentary behavior in early childhood education and care services: A systematic review. Preventive Medicine, 89, 129-139.

Tucker, P., \& Gilliland, J. (2007). The effect of season and weather on physical activity: A systematic review. Public Health, 121(12), 909-922.

Tucker, P., van Zandvoort, M. M., Burke, S. M., \& Irwin, J. D. (2011). Physical activity at daycare: Childcare providers' perspectives for improvements. Journal of Early Childhood Research, 9(3), 207-219. https://doi.org/10.1177/1476718X10389144.

Ulset, V., Vitaro, F., Brendgen, M., Bekkhus, M., \& Borge, A. I. H. (2017). Time spent outdoors during preschool: Links with children's cognitive and behavioral development. Journal of Environmental Psychology, 52(Supplement C), 69-80.

van Zandvoort, M., Tucker, P., Irwin, J. D., \& Burke, S. M. (2010). Physical activity at daycare: Issues, challenges and perspectives. Early Years, 30(2), 175-188.

Wang, C., Hatzigianni, M., Shahaeian, A., Murray, E., \& Harrison, L. J. (2016). The combined effects of teacher-child and peer relationships on children's social-emotional adjustment. Journal of School Psychology, 59, 1-11.

Ward, D. S. (2010). Physical activity in young children: The role of child care. Medicine and Science in Sports and Exercise, 42(3), 499-501. 